\title{
New Trends in Tinnitus Management
}

\author{
Alessandra Fioretti*, Alberto Eibenstein and Marco Fusetti
}

Surgical Sciences Department, ENT, University of L'Aquila, L'Aquila, Italy

\begin{abstract}
Tinnitus is a perception of sound in absence of sound stimulation. Tinnitus in many cases cannot be eliminated by conventional medical treatment with drugs or surgery. Some people who begin to notice tinnitus, whether spontaneous or induced by noise, trauma or other insult, will experience spontaneous resolution, but many patients will have persistent tinnitus. For some of them, tinnitus sensation will be joined by tinnitus suffering, with many adverse effects like anxiety, depression and sleep disorders. For these tinnitus sufferers the psychological and acoustic approach proposed by the Tinnitus Retraining Therapy and Acoustic Desensitization Protocol may be helpful. Periodically new treatments are suggested like low-frequency repetitive transcranial magnetic stimulation and sequential phase shift sound cancellation treatment based on the frequency and loudness matching of the tinnitus. The aim of this work is to review modern considerations for the treatment of tinnitus.
\end{abstract}

Keywords: Tinnitus, hyperacusis, cochlea.

\section{INTRODUCTION}

Tinnitus is defined as a perception of a sound without an external acoustic source. People with tinnitus often describe it as a perception of ringing, whistling or buzzing in one or both ears. It can be persistent, intermittent, or throbbing, depending on the cause. It is important to distinguish between subjective and objective tinnitus. Subjective tinnitus has a neurophysiological origin whereas objective tinnitus can be generated from vascular, muscular or respiratory sources and also from the temporo-mandibular joint. There are many hypotheses to explain the origin of tinnitus even if none has yet been proven. Most of the authors consider the origin of tinnitus as cochlear (lesions of the ciliated cells of the inner ear after acoustic trauma, presbyacusis, sudden hearing loss, etc.) and many patients suffer from hearing loss not subjectively felt. The central processes, especially anxiety, exacerbate the discomfort and whether or not the tinnitus is disturbing, is determined through further central auditory processing of the tinnitus "signal" and its psychological validation. The $10 \%$ of the adult population is afflicted by tinnitus and in many cases consequential changes in quality of life and substantial modifications of behaviour, including anxiety, irritability, depression and sleep disturbances are reported. The failure of many treatments and drugs in particular, has led several teams to propose treatments based on different concepts. Tinnitus with very similar physical characteristics can be perceived as intolerable by some patients but remain insignificant for others. It is difficult to assess the consequences of tinnitus on the patients' quality of life. This absence of correlation between physical intensity of tinnitus, its acoustic characteristics and reported discomfort led to the use of questionnaire-based assessment methods like the Tinnitus Handicap Inventory (THI). The THI is a selfadministered questionnaire to evaluate the impact of tinnitus

*Address correspondence to this author at the Surgical Sciences Department, ENT, University of L'Aquila, L'Aquila, Italy;

Tel/Fax: +390645551655; E-mail: bessi76@yahoo.it on the quality of life. The development of patient-based out come measurements is significantly improving our ability to objectify perceived disability and better quantify the impact of tinnitus and its effects, which are largely subjective. Epidemiologic studies show that anxiety and depression exacerbate the discomfort engendered by tinnitus because patients develop a conditioned reflex of focusing on the tinnitus.

The goal of this study was to review the most recent neurophysiological findings and the new trends in the treatment of subjective tinnitus.

\section{NEUROPHYSIOLOGY OF TINNITUS}

The current opinion is that tinnitus is a consequence of modified neural activity in the central auditory system consequential to a peripheral damage in the auditory structures [1]. The pathophysiological mechanism may be a thalamocortical dysrhythmia with a consequent decreased auditory stimulation and surrounding brain area hyperactivity, inducing a topographical reorganization [2] Central theories suggest an increased spontaneous activity associated with tinnitus in the dorsal cochlear nucleus [3] and the inferior colliculus of the brainstem [4,5] (Fig. 1).

The discordant dysfunction theory was proposed by Jastreboff [6] in 1990. This theory postulates that when the cochlea is damaged by excessive noise, drugs exposure or viral infections the outer hair cells $(\mathrm{OHC})$ are damaged first and inner hair cells (IHC) later. IHC provide sound transduction while $\mathrm{OHC}$ amplification of sounds within the cochlea. Cochlear damage due to acoustic trauma changes the spontaneous firing rates of neurons in the dorsal cochlear nucleus (DCN) and hyperactivity can be observed in the DCN. The DCN, as primary acoustic nucleus and a site of integration of acoustic and sensory inputs, can be an important brain centre for the generation and modulation of tinnitus [7]. One hypothesis is that normal electrical activity in the auditory system becomes pathologically persistent due to persistent changes in the synaptic strength between different neurons in 
the DCN that receive excitation from IHC but not from the damaged $\mathrm{OHC}$.

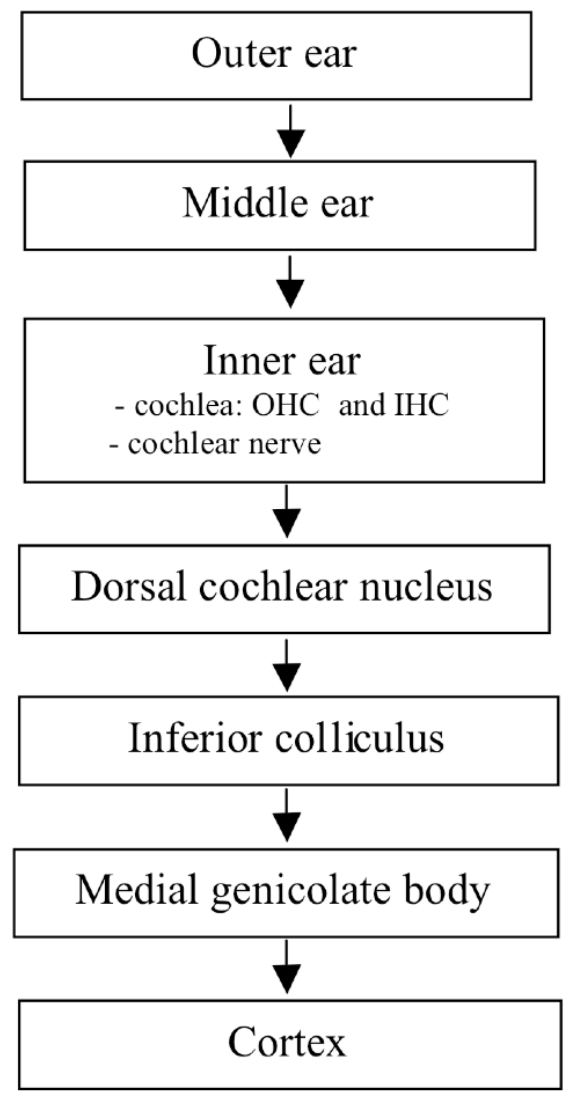

Fig. (1). Sites of tinnitus generation in the auditory pathway. OHC outer hair cells, IHC inner hair cells.

A longstanding hypothesis is that tinnitus and hyperacusis, are triggered by a distinctive hyperfunction of the $\mathrm{OHC}$, originating from reduced or ineffective efferent control in the auditory pathway. The lack of control subsequently leads to altered neural activity in the central auditory pathway. This can be verified by registration of distortion products of otoacoustic emissions (DPOAE). Electrophysiological recordings in the mammalian auditory system showed an increase in neural responsiveness to external sound and a reorganisation of the tonotopic map [8]. Animals exposed to noise trauma show a synchronous neural activity in tonotopically reorganized primary auditory cortex consequent on hearing loss. Diminished intracortical inhibition in the reorganized region appears to enable synchronous network activity that develops when deafferented neurons begin to respond to input via their lateral connections. In humans with tinnitus and hearing loss, this process may generate a phantom sound that is perceived in accordance with the location of the affected neurons in the cortical place map. This neural mechanism of tinnitus [9] assumes that the tonotopic expansion directly causes the phantom sound because deafferentation of a portion of the cochlea reduces the input to the corresponding portion of the tonotopic map in each field. In many cases the area of the cochlea with the largest differences between damaged $\mathrm{OHC}$ and normal IHC may correspond to the slope of hearing loss on the audiogram and the perceived pitch of tinnitus [10]. Further studies are necessary to support this neural mechanism of tinnitus.
The concept of neurovascular dysfunction and neurodegeneration in patients with subjective idiopathic tinnitus has been recently proposed and reported [11].

A biochemical theory was proposed based on the connections between the IHC and auditory nerve mediated by neurotransmitters. The most common excitatory neurotransmitter is glutamate while the primary inhibitory neurotransmitter is GABA. When IHC are damaged excessive glutamate is released with excitotoxicity.

Many people receive high doses of salicylate and a related molecular mechanism in the origin of tinnitus from the periphery of the auditory system is actually proposed. Salicylate has neural excitatory effect on the synapses between the sensory IHC and the dendrites of the cochlear spiral ganglion neurons which express NMDA receptors. Salicylate inhibits cochlear cyclooxygenase whith increased levels of arachidonate which enables NMDA responses to the glutamate that IHC spontaneously release [12]. More studies on neurotransmitters are needed to determine their role in tinnitus development.

A recent research suggests to investigate all the causes of hemodynamic imbalance and autonomic dysregulation which could be responsible of a circulatory impairment of the labyrinth and consequently of tinnitus [13].

\section{NEUROIMAGING OF TINNITUS}

Neuroimaging studies have been used to evidence the cortical expansion and reorganization in tinnitus patients and the relationship between sensory, cognitive and affective systems. In a cross-sectional study of 2193 subjects aged 4182 years studied with brain MRI the authors demonstrated that cerebral infarctions of the basal ganglia $(\mathrm{OR}=0.542)$, thalamus $(\mathrm{OR}=0.441)$, and pons $(\mathrm{OR}=0.319)$ were especially associated with tinnitus [14]. In a study a 3D fast imaging employing steady-state acquisition (3D-FIESTA) sequence was performed in addition to the temporal MR imaging to investigate the role of the vascular loop and compression of the vestibulocochlear nerve at the cerebellopontine angle in patients with unexplained tinnitus. No statistically significant differences were found between the patient and control groups [15].

The key role of inferior colliculus in unilateral subjective tinnitus has been demonstrated using fMRI. The response to sound in the inferior colliculus was elevated in tinnitus patients compared with controls without tinnitus [16].

A neuronal activation in the inferior colliculi and auditory cortices of rats during salicylate-induced tinnitus is also confirmed in a study conducted using positron emission tomography (PET) imaging. These results suggest that microPET imaging might be used to study the effects of drugs to treat tinnitus [17]. Recently Landgrebe et al. described structural alterations in the central nervous system detected in tinnitus patients by voxel-based morphometry (VBM): significant grey matter decreases in the right inferior colliculus and in the left hippocampus confirm the important role of the limbic system in the pathophysiology of tinnitus [18].

\section{DIAGNOSTIC ASSESSMENT}

Unfortunately all these efforts in understanding tinnitus and its treatment have been limited by an inability to objec- 
tively assess this disorder and its sequelae. Standard audiologic tests include audiometry for pure tones and speech, impedence testing, brain auditory evoked potentials (BAEP) and otoacoustic emissions (OAE). Pure tone audiometry is the standard test used to assess the hearing threshold for the frequencies from 125 to $8.000 \mathrm{~Hz}$ (to $16.000 \mathrm{~Hz}$ in highfrequency pure tone audiometry). Speech audiometry is useful in setting hearing aids. BAEP are used to study the pathology of the acoustic nerve and to assess the auditory pathways up to inferior colliculus before MRI investigation. BAEP are suggested in monolateral hearing loss with pathologic responses in cochlear damage, traumatic hearing loss or nerve compression. OAE are registered by an earplug-like device with a microphone which measures the cochlea's response to sound. For this reason OAE represent the cochlear mechanical activity of $\mathrm{OHC}$ and one source of tinnitus may be related to dysfunctions in cochlear mechanical activity.

Specialized audiometric tests like pitch and loudness matching, residual inhibition (RI), minimum masking level (MML) and loudness discomfort level (LDL) are used to find out the subjective qualities of tinnitus. Pitch and loudness matching may be useful to characterize tinnitus frequency and intensity. MML is the difference between the threshold of tinnitus and the lowest intensity to mask the tinnitus. RI is measured as partial or full inhibition of tinnitus after 60 seconds of application of MML $+10 \mathrm{~dB}$. The measurement of LDL is indicated in patients with reduced sound tolerance. These psychophysical measurements have a limited value due to the variability of tinnitus loudness and the poor correlation between tinnitus loudness and tinnitus distress but are useful in counseling and monitoring outcomes. An initial subjective survey of tinnitus characteristics specifically analyzed for duration, time of awareness and presence of hyperacusis (reported as sound intolerance) is strongly recommended. Hyperacusis is defined as abnormal increased sound-induced activity within the auditory pathways.

The use of broadly validated scales allows comparisons between patients and even between institutions. Among the different tinnitus-specific self-assessment questionnaires, the Tinnitus Handicap Inventory (THI) is desirable for its easy administration, incorporation of functional as well as emotional subscales, good validity, strong internal consistency and test-retest reliabilities. The THI is a 25 -item survey that provides a total score and 3 subscale (functional, emotional and catastrophic). The reliability and validity of the Italian version of the Tinnitus Handicap Inventory (THI) is wellestablished [19,20].

\section{NEW TRENDS IN TINNITUS THERAPY}

Various treatments have been proposed for tinnitus therapy with unsuccessful or unproven results. Researches in the pathogenesis of tinnitus suggest that several sites are involved, from the peripheral to central auditory system including the cochlea, neurotransmitters and receptors, ion channels [21].

\section{Surgical Therapy}

Cochlear Implant (CI) is a surgically implanted electronic device for the standard treatment in patients with severe to profound bilateral hearing loss. CI have been noted to reduce tinnitus in some patients [22]. The tinnitus is reduced and masked when the cochlear implant is switched on. Another mechanism of tinnitus improvement may be positive effect of the reorganization of the central auditory nervous system after restoration of peripheral sensory input [23,24]. Residual inhibition has not been regularly demonstrated in sufferers having cochlear implants. Some patients also developed tinnitus for the first time after the CI has been implanted [2527].

\section{Medications}

Anti-seizure medications (like gabapentin) [28,29] and benzodiazepines (like diazepam, lorazepam, alprazolam) [30] activate GABA receptors that will inhibit the continuous firing caused by glutamate. Several trials examined the effect of gabapentin on tinnitus demonstrating no effect [31]. Bauer et al. demonstrated an improvement in patients with tinnitus secondary to acoustic trauma treated with gabapentin [32]. Benzodiazepines could suppress tinnitus by reducing neural activity in the inferior colliculus [33]. These drugs are helpful in reducing anxiety and the sound level of tinnitus but cause drowsiness and can lead to dependence.

Medications that are antagonistic to glutamate and its receptor N-methyl-d-aspartate (NMDA) (like caroverine and memantine) reduces glutamate-induced continuous firing of the neuron but they also have been shown to exhibit hallucinogenic properties [34].

Memantine, an antiglutamatergic drug, is not efficacy in the treatment of tinnitus [35].

Caroverine acts as a glutamate antagonist. Denk et al. [36] reported variable results in patients with tinnitus treated with caroverine. Ehrenberger et al. [37] investigated the effects of Caroverine on tinnitus patients for several years. Initially they had success in $63 \%$ of participants using an IV infusion of caroverine subsequently they reported a success in $50 \%$ of patients with Meniere disease and sudden hearing loss.

Acamprosate has been recently used like a glutamate antagonist. A small clinical trial of acamprosate conducted on 25 tinnitus patients showed that $87 \%$ had some degree of relief and nearly $48 \%$ had a reduction of more than $50 \%$. Many side effects are described (depression, diarrhea, anxiety) and the drowsiness caused by acamprosate itself may have contributed to improvement as many tinnitus patients have trouble sleeping [38].

Ginkgo Biloba Extract, is a powerful glutamate antagonist which acts as a strong anti-oxidant within the cochlea, helping to minimize damage caused by free radical build-up but not proven results are demonstrated in tinnitus treatment.

Studies with tricyclic antidepressant agents (amitriptyline nortriptyline and trimipramine) and a selective serotonin reuptake inhibitor antidepressant (paroxetine) didn't prove the efficacy of these agents in the management of tinnitus $[39,40]$. Neramexane is currently in phase III clinical trials for the treatment of subjective tinnitus.

\section{Physiological Treatments}

\section{Transcranial Magnetic Stimulation}

A growing number of studies demonstrate reduction of tinnitus after repeated sessions of low-frequency repetitive 
transcranial magnetic stimulation (rTMS). rTMS produces a neurostimolation of specific brain regions potentially involved in the pathophysiology of tinnitus. rTMS might represent a new promising approach for the treatment of tinnitus based on the involvement of dysfunctional neuroplastic processes in the brain. TMS may modulate the hyperexcitability of neurons in cerebral cortex that is associated with some forms of tinnitus. Better results with rTMS have been seen in patients with normal hearing and tinnitus of shorter duration ( $<4$ years). The treatment consists in 10 sessions of rTMS over the left temporal or left temporoparietal cortex within two weeks. rTMS is to avoid in presence of epilepsy, pregnancy, cardiac pace-maker, electrical implants. rTMS is a non-invasive method which has proven effective in auditory hallucinations [41-45]. Plewnia et al. [46] demonstrated a reduction of tinnitus loudness after temporo-parietal PETguided low-frequency rTMS in nine patients with chronic tinnitus. These results underline the key role of higher-order sensory processing in the pathophysiology of chronic tinnitus. Marcondes et al. also demonstrated in patients with tinnitus a significant reduction of neural activity in the inferior temporal cortex measured by SPECT after active rTMS [47].

\section{Other Approaches}

\section{Low-Level Laser Therapy}

Transmeatal, low laser laser therapy (LLLT) is performed with red $(630 \mathrm{~nm})$ or near infrared $(830 \mathrm{~nm})$ laser light applied through the external ear conduct. It was found to be useful for the treatment of chronic tinnitus. It has been assumed that LLLT is capable of increasing cell proliferation, synthesis of ATP, promoting the local blood flow in the inner ear and activating repair mechanisms in the inner ear [48]. Other clinical studies didn't demonstrate a significant efficacy of LLLT in tinnitus treatment [49].

\section{Phase-Out Treatment}

The sequential phase shift sound cancellation treatment is recommended for patients with pure tone tinnitus at a constant frequency. A computer program processed a sound, based on the frequency and loudness matching of the tinnitus, that interferes with the tinnitus and analyzes the amplitude and phase of the background noise so that the tinnitus isn't heard [50]. Vermeire et al. [51] demonstrated positive effects in $60 \%$ of treated patients. A multicenters study on 493 patients treated with phase-shift sound wave cancellation for treatment of predominant tone tinnitus reports a reduction in tinnitus volume (defined as $>/=6 \mathrm{~dB}$ ) in $49-72$ per cent of patients [52]. The hypothesis is that using a tinnitus patient's self-assessed pitch and volume wave, shifted sequentially $6^{\circ}$ for 30 seconds (over 30 minutes), would cancel the neuronal complex in the auditory cortex responsible for predominant tone tinnitus. Further studies are necessary to validate this treatment.

\section{Neuromonics}

The Neuromonics tinnitus treatment is designed to interact and desensitize tinnitus disturbance using a customized sound therapy in a framework of structured counselling $[53,54]$. The treatment utilizes a spectrally-modified neural stimulus in the form of music and noise, customized for each individual's audiometric profile. In a recent study $90 \%$ of patients reported a reduction in their tinnitus-related distur- bance of $40 \%$ and more and after six months of treatment $80 \%$ of subjects reported a significant improvement of tinnitus disturbance [55]. Neuromonics is not actually available in Europe but only in Australia and USA.

Recent studies have indicated that tinnitus-related activity leads to changes in tonotopic representation in auditory cortex rather than aberrant activity in the periphery. Auditory discrimination training (ADT) could partially reverse the changes in tonotopic reorganization in the central nervous system after a peripheral damage and improve tinnitus [56,57].

\section{Cognitive-Behavioral Therapy (CBT) and Tinnitus Retraining Therapy (TRT)}

The influence attributed to the limbic and autonomic nervous systems on how tinnitus is experienced has led to the development of techniques of habituation, widely used in cognitive-behavioral therapy (CBT) and in Tinnitus Retraining Therapy (TRT).

The aim of CBT is to modify harmful behaviours and thoughts by using "deconditioning" techniques. The main objectives of CBT to obtain the habituation to tinnitus are reducing arousal levels through relaxation therapy and changing negative thoughts about tinnitus through cognitive therapy. Londero et al. reported that $75 \%$ of the patients showed improvement on assessment one year after CBT ended [58]. Goebel et al. confirm the long-term benefits of CBT for tinnitus [59]. Presently, mindfulness meditation offers a new psychological treatment for tinnitus patients [60].

Several studies have confirmed the usefulness of TRT in the management of patients with tinnitus [61-76]. TRT is possible and helpful to patients with disabling chronic tinnitus.

The goal of TRT is to remove the perception of tinnitus from the patient's consciousness by method aimed at habituation of the reaction with directive counselling and the perception of the tinnitus signal with the sound therapy. The aim of counselling is to actively change the way the patient thinks about tinnitus and reverse the distress produced by tinnitus removing the negative association given to the tinnitus signal (habituation of the reaction). Wearable low-level broadband noise generators are used in sound therapy to facilitate habituation of perception. They do not produce a masking signal but are regulated at the mixing-point to decrease the contrast between the tinnitus signal and random background activity within the neural pathways.

\section{CONCLUSION}

It is difficult to study and treat tinnitus because of the lack of objective tools to quantify and measure it. In these last years the use of PET/SPECT and functional magnetic resonance imaging permit to identify areas of aberrant neural activity in the human brain associated with the perception of tinnitus. To date, there has been no therapeutically successful treatment of tinnitus in terms of medium or long term remission. New insights in the neural basis of tinnitus may be found with the development of animal models and advanced electrophysiological recording techniques to study behavioral assessment and therapeutic compounds to treat tinnitus. Some authors showed that many patients have 
somatic tinnitus whereby movements manipulations of the eyes, head, neck, jaw and shoulder can modulate the loudness and pitch of their tinnitus [77-82]. These findings may underline the involvement of non auditory centers in the pathogenesis and regulation of tinnitus. A future goal will be to reach the consensus both for patient assessments and for outcome measurements. Longer and multi-institutional studies should confirm this trend towards better cooperation between research centres.

\section{REFERENCES}

[1] Eggermont JJ, Roberts LE. The neuroscience of tinnitus. Trends Neurosci 2004; 27: 676-682

[2] De Ridder D, De Mulder G, Verstraeten E, et al. Auditory cortex stimulation for tinnitus. Acta Neurochirur Suppl 2007; 97: 451-62

[3] Kaltenbach JA. Neurophysiologic mechanisms of tinnitus. J Am Acad Audiol. 2000; 11: 125-37

[4] Melcher JR, Sigalovsky IS, Guinan JJ Jr, Levine RA. Lateralized tinnitus studied with functional magnetic resonance imaging: abnormal inferior colliculus activation. J Neurophysiol 2000; 83: 1058-72;

[5] Melcher JR, Levine RA, Bergevin C, Norris B. The auditory midbrain of people with tinnitus: abnormal sound-evoked activity revisited. Hear Res 2009; 257: 63-74

[6] Jastreboff PJ. Phantom auditory perception (tinnitus): mechanisms of generation and perception. Neurosci Res 1990; 8: 221-54

[7] Kaltenbach JA, Godfrey DA. Dorsal cochlear nucleus hyperactivity and tinnitus: Are they related? Am J Audiol 2008; 17: S148-61.

[8] Salvi RJ, Lockwood AH, Burkard R. Neural plasticity and tinnitus. In: Tyler RS, Ed. San Diego, CA: Tinnitus Handbook Singular 123-48.

[9] Hazell JW, Jastreboff PJ. Tinnitus. Auditory mechanisms: a model for tinnitus and hearing impairment. J Otolaryngol 1990; 19: 1-5.

[10] Norena AJ, Micheyl C, Chery-Croze S, Collet L. Psychoacoustic characterization of the tinnitus spectrum: implications for the underlying mechanisms of tinnitus. Audiol Neurootol 2002; 7: 35869.

[11] Shulman A, Goldstein B, Strashun AM. Central nervous system neurodegeneration and tinnitus: a clinical experience. Part II: translational neurovascular theory of neurodegenerative CNS disease and tinnitus. Int Tinnitus J 2008; 14: 43-51.

[12] Ruel J, Chabbert C, Nouvian R, et al. Salicylate enables cochlear arachidonic-acid-sensitive NMDA receptor responses. J Neurosci 2008; 28: 7313-23.

[13] Pirodda A, Brandolini C, Ferri GG, Modugno GC, Esposti DD, Borghi C. Possible influence on heart rate on tinnitus. Med Hypotheses 2009; 72: 45-6.

[14] Sugiura S, Uchida Y, Nakashima T, Yoshioka M, Ando F, Shimokata H. Tinnitus and brain MRI findings in Japanese elderly. Acta Otolaryngol 2008; 128: 525-529.

[15] Gultekin S, Celik H, Akpek S, Oner Y, Gumus T, Tokgoz N. Vascular Loops at the Cerebellopontine Angle: Is There a Correlation with Tinnitus?AJNR Am J Neuroradiol 2008; 29: 1746-69.

[16] Lanting CP, De Kleine E, Bartels H, Van Dijk P. Functional imaging of unilateral tinnitus using fMRI. Acta Otolaryngol 2008; 128: 415-21.

[17] Paul AK, Lobarinas E, Simmons R, et al. Metabolic imaging of rat brain during pharmacologically-induced tinnitus. Neuroimage 2009; 44: 312-8

[18] Landgrebe M, Langguth B, Rosengarth K, et al. Structural brain changes in tinnitus: grey matter decrease in auditory and nonauditory brain areas. Neuroimage 2009; 46: 213-8.

[19] Passi S, Ralli G, Capparelli E, Mammone A, Scacciatelli D, Cianfrone $\mathrm{G}$. The THI questionnaire: psychometric data for reliability and validity of the Italian version. Int Tinnitus J 2008; 14: 26-33.

[20] Monzani D, Genovese E, Marrara A, et al. Validity of the Italian adaptation of the Tinnitus Handicap Inventory; focus on quality of life and psychological distress in tinnitus-sufferers. Acta Otorhinolaryngol Ital 2008; 28: 126-34.

[21] Baguley DM. What progress have we made with tinnitus? The Tonndorf lecture 2005. Acta Otolaryngol Suppl 2006; 556: 4-8.

[22] Baguley DM, Atlas MD. Cochlear implants and tinnitus. Prog Brain Res 2007; 166: 347-55.
[23] Moller AR. Pathophysiology of tinnitus. Otolaryngol Clin North Am 2005; 36: 249-66

[24] Del Bo L, Ambrosetti U. Hearing aids for the treatment of tinnitus. Prog Brain Res 2007; 166: 341-5.

[25] Van de Heyning P, Vermeire K, Diebl M, Nopp P, Anderson I, De Ridder D. Incapacitating unilateral tinnitus in single-sided deafness treated by cochlear implantation. Ann Otol Rhinol Laryngol 2008; 117: 645-52

[26] Yonehara E, Mezzalira R, Porto PR, et al. Can cochlear implants decrease tinnitus? Int Tinnitus J 2006; 12: 172-4

[27] Quaranta N, Fernandez-Vega S, D'elia C, Filipo R, Quaranta A. The effect of unilateral multichannel cochlear implant on bilaterally perceived tinnitus. Acta Otolaryngol 2008; 128: 159-63.

[28] Piccirillo JF, Finnell J, Vlahiotis A, Chole RA, Spitznagel E Jr. Relief of idiopathic subjective tinnitus: is gabapentin effective? Arch Otolaryngol Head Neck Surg 2007; 133: 390-97.

[29] Witsell DL, Hannley MT, Stinnet S, Tucci DL. Treatment of tinnitus with gabapentin: a pilot study. Otol Neurotol 2007; 28: 11-5

[30] Johnson RM, Brummett R, Schleuning A. Use of alprazolam for relief of tinnitus. A double-blind study. Arch Otolaryngol Head Neck Surg 1993; 119: 842-45.

[31] Bakhshaee M, Ghasemi M, Azarpazhooh, et al. Gabapentin effectiveness on the sensation of subjective idiopathic tinnitus: a pilot study. Eur Arch Otorhinolaryngol 2008; 265: 525-30.

[32] Bauer CA, Brozoski TJ. Effect of gabapentin on the sensation and impact of tinnitus. Laryngoscope 2006; 116: 675-81

[33] Szczepaniak WS, Moller AR. Effects of baclofen, clonazepam and diazepam on tone exposure induced hyperexcitability of the inferior colliculus in the rat: possible therapeutic implications for pharmacological management of tinnitus and hyperacusis. Hearing Research 1996; 97: 46-53.

[34] Lobarinas E, Yang G, Sun W, et al. Salicylate- and quinineinduced tinnitus and effects of memantine. Acta Otolaryngol Suppl 2006; 556: 13-9.

[35] Figuerido RR, Langguth B, Mello de Oliveira P, Aparecida de Azevedo A. Tinnitus treatment with memantine. Otolaryngol Head Neck Surg 2008; 138: 492-6

[36] Denk DM, Heinzl H, Franz P, Ehrenberger K. Caroverine in tinnitus treatment. A placebo-controlled blind study. Acta Otolaryngol 1997; 117: 825-30

[37] Ehrenberger K. Topical administration of Caroverine in somatic tinnitus treatment: proof-of-concept study. Int Tinnitus J 2005; 11: 34-7.

[38] Azevedo AA, Figuerido RR. Tinnitus treatment with acamprosate: double-blind study. Braz J Otorhinolaryngol 2005; 71: 618-23.

[39] Baldo P, Doree C, Lazzarini R, Molin P, McFerran D. Antidepressants for patients with tinnitus. Cochrane Database of Systematic Reviews 2006; Issue 4. Art. No.: CD003853.

[40] Robinson SK, Viirre ES, Stein MB. Antidepressant therapy in tinnitus. Hear Res 2007; 226: 221-31.

[41] Langguth B, Hajak G, Kleinjung T, Pridmore S, Sand P, Eichhammer P. Repetitive transcranial magnetic stimulation and chronic tinnitus. Acta Otolaryngol Suppl 2006; 556: 102-5.

[42] Kleinjung T, Eichhammer P, Landgrebe M, et al. Combined temporal and prefrontal transcranial magnetic stimulation for tinnitus treatment: a pilot study. Otolaryngol Head Neck Surg 2008; 138 : 497-501.

[43] Mennemeier M, Chelette KC, Myhill J, et al. Maintenance repetitive transcranial magnetic stimulation can inhibit the return of tinnitus. Laryngoscope 2008; 118: 1228-32.

[44] Smith JA, Mennemeier M, Bartel T, et al. Repetitive transcranial magnetic stimulation for tinnitus: a pilot study. Laryngoscope. 2007; 117: 529-34.

[45] Kleinjung T, Vielsmeier V, Landgrebe M, Hajak G, Langguth B. Transcranial magnetic stimulation: a new diagnostic and therapeutic tool for tinnitus patients. Int Tinnitus J 2008; 14: 112-8.

[46] Plewnia C, Reimold M, Najib A, Reischl G, Plontke SK, Gerloff C. Moderate therapeutic efficacy of positron emission tomographynavigated repetitive transcranial magnetic stimulation for chronic tinnitus: a randomised, controlled pilot study. J Neurol Neurosurg Psychiatry 2007; 78: 152-6.

[47] Marcondes RA, Sanchez TG, Kii MA, et al. Repetitive transcranial magnetic stimulation improve tinnitus in normal hearing patients: a double-blind controlled, clinical and neuroimaging outcome study. Eur J Neurol 2010; 17: 38-44. 
[48] Gungor A, Dogru S, Cincik H, Erkul E, Poyrazoglu E. Effectiveness of transmeatal low power laser irradiation for chronic tinnitus. J Laryngol Otol 2008; 122: 447-51.

[49] Teggi R, Bellini C, Piccioni LO,Palonta F, Bussi M. Transmeatal low-level laser therapy for chronic tinnitus with coclea dysfunction. Audiol Neurootol 2009; 14: 115-120.

[50] Lipman RI, Lipman SP. Phase-shift treatment for predominant tone tinnitus. Otolaryngol Head Neck Surg 2007; 136: 763-8

[51] Vermeire K, Heyndrickx K, De Ridder D, Van de Heyning P. Phase-shift tinnitus treatment: an open prospective clinical trial. BENT 2007; 3(Suppl 7): 65-9.

[52] Choy DS, Lipman RA, Tassi GP. Worldwide experience with sequential phase-shift sound cancellation treatment of predominant tone tinnitus. J Laryngol Otol 2010; 124: 366-9

[53] Davis PB, Wilde RA, Steed LG, Hanley PJ. Treatment of tinnitus with a customized acoustic neural stimulus: a controlled clinical study. Ear Nose Throat J 2008; 87: 330-9.

[54] Hanley PJ, Davis PB. Treatment of tinnitus with a customized, dynamic acoustic neural stimulus: underlying principles and clinical efficacy. Trends Amplif 2008; 12: 210-22

[55] Davis PB, Paki B, Hanley PJ. Neuromonics Tinnitus Treatment: third clinical trial. Ear Hear 2007; 28: 242-59.

[56] Herraiz C, Diges I, Cobo P, Aparicio JM. Cortical reorganisation and tinnitus: principles of auditory discrimination training for tinnitus management. Eur Arch Otorhinolaryngol 2009; 266: 9-16.

[57] Pineda JA, Moore FR, Viirre E. Tinnitus treatment with customized sounds. Int Tinnitus J 2008; 14: 17-25.

[58] Londero A, Peignard P, Malinvaud D, Avan P, Bonfils P. Acouphènes et thérapie comportementale et cognitive. Presse Med 2006; 35: 1213-21.

[59] Goebel G, Kahl M, Arnold W, Fichter M. 15 years prospective follow-up study of behavioural therapy in a large samplke of inpatients with chronic tinnitus. Acta Otolaryngol 2006; 126: 70-9.

[60] Sadler M, Stephens SDG, Kennedy V. Tinnitus rehabilitation: a mindfulness meditation cognitive behaviour therapy approach. J Laryngol Otol 2008; 122: 31-7.

[61] Jastreboff PJ, Jastreboff MM. Tinnitus retraining therapy (TRT) as a method for treatment of tinnitus and hyperacusis patients. J Am Acad Audiol 2000; 11: 162-77.

[62] Jastreboff PJ, Jastreboff MM. Tinnitus retraining therapy for patients with tinnitus and decreased sound tolerance. Otolaryngol Clin North Am 2003; 36: 321-36.

[63] Jastreboff PJ, Hazell JW. Tinnitus retraining therapy: implementing the neurophysiological model. New York: Cambridge University Press 2007.

[64] Jastreboff PJ. Tinnitus retraining therapy. Prog Brain Res 2007; 166: 415-23

[65] Holdefer L, Oliveira CA, Venosa AR. Group therapy for patients with tinnitus at the University of Brasilia Medical School. Braz J Otorhinolaryngol 2010; 76: 102-6.

[66] Mazurek B, Fischer F, Haupt H, Georgiewa P, Reisshauer A, Klapp BF. A modified version of tinnitus retraining therapy: observing long-term outcome and predictors. Audiol Neurootol 2006; 11: $276-286$.
[67] Madeira G, Montmirail Ch, Decat M, Gersdorff M. TRT: results after one year treatment. Rev Laryngol Otol Rhinol 2007; 128: $145-8$.

[68] Koizumi T, Nishimura T, Sakaguchi T, Okamoto M, Hosoi H. Estimation of factors influencing the results of tinnitus retraining therapy. Acta Otolaryngol Suppl 2009; (562): 40-5.

[69] Seydel C, Haupt H, Szczepek AJ, Klapp BF, Mazurek B. Longterm improvement in tinnitus after modified tinnitus retraining therapy enhanced by a variety of psychological approaches. Audiol Neurootol 2010; 15: 69-80

[70] Forti S, Costanzo S, Crocetti A, Pignataro L, Del Bo L, Ambrosetti $\mathrm{U}$. Are results of tinnitus retraining therapy maintained over time? 18-month follow-up after completion of therapy. Audiol Neurootol 2009; 14: 286-9.

[71] Hatanaka A, Ariizumi Y, Kitamura K. Pros and cons of tinnitus retraining therapy. Acta Otolaryngol 2008; 128: 365-8.

[72] Herraiz C, Hernandez FJ, Plaza G, de los Santos G. Long-term clinical trial of tinnitus retraining therapy. Otolaryngol Head Neck Surg 2005; 133: 774-9.

[73] Herraiz C, Hernandez FJ, Toledano A, Aparicio JM. Tinnitus retraining therapy: prognosis factors. Am J Otolaryngol 2007; 28: 225-9.

[74] Henry JA, Jastreboff MM, Jastreboff PJ, Schechter MA, Fausti SA. Assessment of patients for treatment with tinnitus retraining therapy. J Am Acad Audiol 2002; 13: 523-544.

[75] Henry JA, Schechter MA, Zaugg TL, et al. Clinical trial to compare tinnitus masking and tinnitus retraining therapy. Acta Otolaryngol Suppl 2006; (556): 64-9.

[76] Henry JA, Loovis C, Montero M, et al. Randomized clinical trial: group counseling based on tinnitus retraining therapy. J Rehabil Res Dev 2007; 44: 21-32.

[77] Sanchez TG, Guerra GC, Lorenzi MC, Brandão AL, Bento RF. The influence of voluntary muscle contractions upon the onset and modulation of tinnitus. Audiol Neurootol 2002; 7: 370-5

[78] Sanchez TG, da Silva Lima A, Brandão AL, Lorenzi MC, Bento RF. Somatic modulation of tinnitus: test reliability and results after repetitive muscle contraction training. Ann Otol Rhinol Laryngol 2007; 116: 30-5

[79] Levine RA, Nam EC, Oron Y, Melcher JR. Evidence for a tinnitus subgroup responsive to somatosensory based treatment modalities. Prog Brain Res 2007; 166: 195-207

[80] Latifpour DH, Grenner J, Sjödahl C. The effect of a new treatment based on somatosensory stimulation in a group of patients with somatically related tinnitus. Int Tinnitus J 2009; 15: 94-9.

[81] Vanneste S, Plazier M, Van de Heyning P, De Ridder D. Transcutaneous electrical nerve stimulation (TENS) of upper cervical nerve (C2) for the treatment of somatic tinnitus. Exp Brain Res 2010; PMID: 20505927

[82] Kapoula Z, Yang Q, Vernet M, Bonfils P, Londero A. Eye movement abnormalities in somatic tinnitus: fixation, smooth pursuit and optokinetic nystagmus. Auris Nasus Larynx 2010; 37: 314-21.

(C) Fioretti et al.; Licensee Bentham Open.

This is an open access article licensed under the terms of the Creative Commons Attribution Non-Commercial License (http://creativecommons.org/licenses/by-nc/3.0/) which permits unrestricted, non-commercial use, distribution and reproduction in any medium, provided the work is properly cited. 